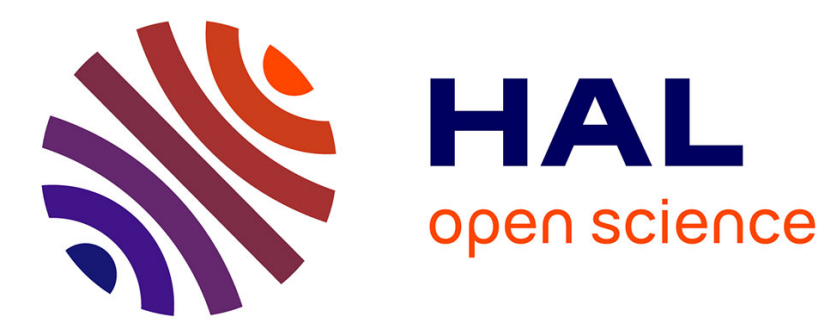

\title{
Gas flow in plant microfluidic networks controlled by capillary valves
}

Marie Capron, Philippe Tordjeman, François Charru, Eric Badel, Hervé Cochard

\section{- To cite this version:}

Marie Capron, Philippe Tordjeman, François Charru, Eric Badel, Hervé Cochard. Gas flow in plant microfluidic networks controlled by capillary valves. Physical Review E: Statistical, Nonlinear, and Soft Matter Physics, 2014, 89 (3), 10.1103/PhysRevE.89.033019 . hal-01189937

\section{HAL Id: hal-01189937 \\ https://hal.science/hal-01189937}

Submitted on 1 Sep 2015

HAL is a multi-disciplinary open access archive for the deposit and dissemination of scientific research documents, whether they are published or not. The documents may come from teaching and research institutions in France or abroad, or from public or private research centers.
L'archive ouverte pluridisciplinaire HAL, est destinée au dépôt et à la diffusion de documents scientifiques de niveau recherche, publiés ou non, émanant des établissements d'enseignement et de recherche français ou étrangers, des laboratoires publics ou privés. 


\title{
Gas flow in plant microfluidic networks controlled by capillary valves
}

\author{
M. Capron, ${ }^{*}$ Ph. Tordjeman, ${ }^{\dagger}$ and F. Charru \\ Université de Toulouse, INPT-CNRS, Institut de Mécanique des Fluides de Toulouse, Allée du Professeur C. Soula, 31400 Toulouse, France \\ E. Badel and H. Cochard \\ INRA, UMR 547 PIAF, 5 chemin de Beaulieu, F-63039 Clermont-Ferrand, France \\ and Clermont Université, Université Blaise Pascal, UMR A547 PIAF, F-63000 Clermont-Ferrand Cedex 2, France
}

(Received 13 December 2013; published 28 March 2014)

\begin{abstract}
The xylem vessels of trees constitute a model natural microfluidic system. In this work, we have studied the mechanism of air flow in the Populus xylem. The vessel microstructure was characterized by optical microscopy, transmission electronic microscopy (TEM), and atomic force microscopy (AFM) at different length scales. The xylem vessels have length $\approx 15 \mathrm{~cm}$ and diameter $\approx 20 \mu \mathrm{m}$. Flow from one vessel to the next occurs through $\sim 10^{2}$ pits, which are grouped together at the ends of the vessels. The pits contain a thin, porous pit membrane with a thickness of $310 \mathrm{~nm}$. We have measured the Young's moduli of the vessel wall and of the pits (both water-saturated and after drying) by specific nanoindentation and nanoflexion experiments with AFM. We found that both the dried and water-saturated pit membranes have Young's modulus around 0.4 MPa, in agreement with values obtained by micromolding of pits deformed by an applied pressure difference. Air injection experiments reveal that air flows through the xylem vessels when the differential pressure across a sample is larger than a critical value $\Delta P_{c}=1.8 \mathrm{MPa}$. In order to model the air flow rate for $\Delta P \geq \Delta P_{c}$, we assumed the pit membrane to be a porous medium that is strained by the applied pressure difference. Water menisci in the pit pores play the role of capillary valves, which open at $\Delta P=\Delta P_{c}$. From the point of view of the plant physiology, this work presents a basic understanding of the physics of bordered pits.
\end{abstract}

DOI: 10.1103/PhysRevE.89.033019

PACS number(s): 47.61.-k, 87.19.-j

\section{INTRODUCTION}

The study of natural microfluidic systems in plants is motivated not only by our scientific interest in fundamental plant physiology but also by the possibility that a biomimetic approach may lead to the development of novel synthetic microfluidic systems. In biology, fluid motion is generally controlled by flow through membranes and is based on complex physical, chemical, and biological mechanisms. These mechanisms, which appear at the molecular scale, are often coupled and are difficult to distinguish by conventional analytical techniques.

The xylem of trees can be considered as a model microfluidic system. It is composed of cells connected together by a large number of small membranes called "pits" (Fig. 1). The cell size depends on the tree species. For angiosperm trees, the vessel length varies between 1 and $40 \mathrm{~cm}$, with diameters in the range of 10-100 $\mu \mathrm{m}$ [1]. Pits are holes in the secondary cell wall layers closed by a thin, permeable, pit membrane. These membranes are composed of a cellulosic layer and may be covered with pectin. For angiosperms, the typical diameter and thickness of the pit membrane are around $10 \mu \mathrm{m}$ and $400 \mathrm{~nm}$, respectively [2].

According to the cohesion-tension theory, sap flows through the xylem under a tensile force generated by the evaporation of water at the leaf surface [3]. Sap pressures are negative in xylem vessels, typically between $-1 \mathrm{MPa}$ and $-10 \mathrm{MPa}$ [4]. According to the physical model of Wheeler and Stroock [5], the negative pressure is regulated during water transpiration

*mcapron@imft.fr

†philippe.tordjeman@imft.fr by a reduction of capillary pressure within the pores of leaf cells. The sap is thermodynamically metastable under tension, and when the negative pressure reaches a critical value, cavitation occurs. As an example, Vincent et al. [6] showed by studying synthetic trees that cavitation appears at a pressure $\approx-22 \mathrm{MPa}$ for water. If nuclei for cavitation are present, the water cannot withstand such large tensions and the critical pressure $\approx-2 \mathrm{MPa}$ [4]. Just after cavitation, the cavitation bubbles, containing mainly water vapor, grow within the cells by evaporation, thereby increasing the cell pressure. If cavitation has occurred in the cell on one side of a pit membrane but not on the other, a large pressure difference can occur across the pit membrane. If the membrane is unable to contain the gas, then gas can flow from one cell to the next, leading to the embolism of the vessels and, sometimes, the death of the tree.

One controversial hypothesis is that the gas flow in the xylem is passive, from a chemical and biological point of view, and is controlled by capillarity at the length scale of the pit pores [5,7-10]. In this scenario, gas flow occurs if the pressure difference between two adjacent cells, filled with gas and liquid, reaches or exceeds a threshold value given by the Laplace relation [11]:

$$
\Delta P_{c}=\frac{4 \gamma \cos \theta}{d_{\text {pore }}},
$$

where $d_{\text {pore }}$ is the pore diameter, $\gamma$ is the gas-sap surface tension, and $\theta$ is the contact angle between the vapor interface and the pit membrane. The model is based on the idea that a capillary meniscus maintains the pressure difference $\Delta P$ and prevents any gas flow when $\Delta P<\Delta P_{c}$. Pores in the pit membrane act like valves and open (in the presence of 


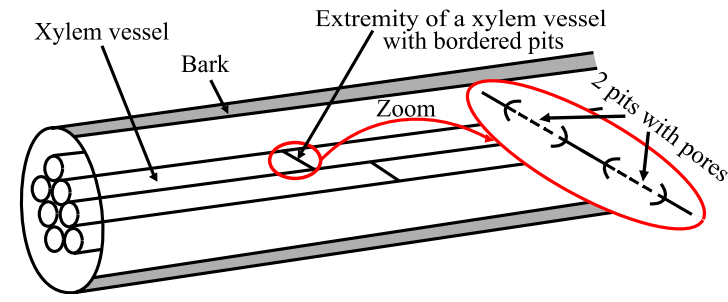

FIG. 1. (Color online) Schema of microfluidics plant networks. The pit field is at the end of the xylem vessel.

gas) only above the critical pressure difference. Thus, $\Delta P_{c}$ is assumed to be a characteristic parameter of the trees that depends on the pit membrane pore structure. However, in practice $\Delta P_{c}$ is also a complex function of the pit membrane mechanical properties which govern the opening of the pore when a pressure difference is applied.

We have investigated the flow of air in Populus wood and have studied how the geometry of the pit membranes is modified by the pressure difference across the membrane. To achieve this, we measured the nanomechanical properties of the individual elements that form the structure of the pits, using atomic force microscopy (AFM). These experiments were performed (in both dry and wet environments) in order to quantify the pit membrane deflection when subjected to a pressure difference.

Pit membrane micromolding experiments were also performed in order to observe directly the deformation of the membranes and thereby verify that we can predict the pit membrane deflection and the opening of the pores at a given pressure difference using mechanical properties measured at the nanoscale. Air injection experiments (called "air seeding" experiments in the botanical literature) were performed to measure the permeability of the xylem to air flow above the threshold capillary pressure difference $\Delta P_{c}$. The results were interpreted by a model that takes the pit membrane mechanical properties, and membrane deformation, into account. This work supports the idea that embolism resistance in trees is controlled by the physical mechanism of capillary valves with properties that depend upon the coupling between structure and material properties at the pit scale.

\section{EXPERIMENTS}

\section{A. Materials}

Branches were collected from Populus deltoides $\times$ Populus nigra during the 2011 growing season from mature trees growing at the PIAF trial grounds (INRA-Clermont Ferrand, France). Branch segments were cut from trees, sealed in plastic bags, brought to the laboratory, and immersed in deionized water. Specimens were studied in either the dried or wetted states. For air seeding experiments, thin branches around $300 \mathrm{~mm}$ long and $5 \mathrm{~mm}$ diameter were used. The sample length was thus higher than the mean length of the xylem vessels $(150 \mathrm{~mm})$ [1]. For AFM experiments, 10-mm square samples of thickness $60 \mu \mathrm{m}$ were cut from lateral branches and trimmed with a razor blade. Two series of measurements were performed, one on dried samples and the other on water-saturated samples. Specimens to be studied dry were cut from the immersed branches and dried during 15 days at room temperature. Specimens to be studied wet were cut from immersed branches and conserved in deionized water for no more than a month before use. When wet specimens were placed on the AFM specimen-holder a small drop of water was deposited on the sample, and the force-displacement curves were measured by plunging the AFM cantilever into the drop. Thus, the samples were never dehydrated.

Before AFM experiments, the samples were observed with a $\times 100$ optical microscope (Zeiss Axio Scope A1) using fluorescent light in order to select the area for AFM measurements. For transmission electron microscopy (TEM), samples were embedded in LR white resin (London Resin Company), which gradually replaced the ethanol. The resin was then polymerized for 2 days at $55^{\circ} \mathrm{C}$. Ultrathin sections were obtained using a diamond-coated blade and stained with uranyl acetate for $10 \mathrm{~min}$ and lead citrate for $1 \mathrm{~min}$. TEM was performed on transverse sections using a JEOL JEM $1210 \mathrm{TEM}$ at $80 \mathrm{keV}$, and the images were analyzed using Image J software (Rasband 1997-2012). For micromolding experiments, segments of branches approximately $150 \mathrm{~mm}$ long and $5 \mathrm{~mm}$ in diameter were used. We carried out two series of measurements at three different injection pressures. In order to visualize the micromolds, the samples were observed with the optical microscope using a DIC light and with a Philips SEM 505 at the Electron Microscopy Laboratory of the Institut National de la Recherche Agronomique, Theix (Clermont-Ferrand, France).

\section{B. Atomic force microscopy experiments \\ 1. Equipment}

The microstructure and nanomechanical properties of the Populus pits were characterized by an AFM with nanometer resolution [12-15]. An Agilent 5500 Scanning Probe Microscope was used with its software PicoView, both in tapping mode for imaging and in contact mode for nanomechanics. The experiments were performed using a scanning probe Nanotools model with ACT tips in tapping mode and a scanning probe MESP Veeco in contact mode. The manufacturer-supplied characteristics of the Nanotools ACT tips (single crystalsilicon) are: tip radius $R=10 \mathrm{~nm}$; cantilever thickness $t=4.5 \mu \mathrm{m}$; cantilever length $l=125 \mu \mathrm{m}$; cantilever width $w=35 \mu \mathrm{m}$; cantilever stiffness $k=25-75 \mathrm{~N} \mathrm{~m}^{-1}$; resonance cantilever frequency $f_{0}=300 \mathrm{kHz}$. The characteristics of the MESP tips [antimony $(n)$ doped $\mathrm{Si}$ ] are: tip radius $R=$ $2 \mathrm{~nm}$; cantilever thickness $t=2.5 \mu \mathrm{m}$; cantilever length $l=$ $200 \mu \mathrm{m}$; cantilever width $w=23-33 \mu \mathrm{m}$; cantilever stiffness $k=1-5 \mathrm{~N} \mathrm{~m}^{-1}$; resonance cantilever frequency $f_{0}=60 \mathrm{kHz}$.

For nanomechanical experiments (MESP tips), the cantilever stiffness and the tip radius are the main parameters required for quantitative measurements of the local values of the material mechanical properties. These parameters should be determined independently of the values given by the manufacturers. First, the calibration factor for the displacement detector $1 / \chi=2.89 \pm 0.14 \times 10^{8} \mathrm{~V} \mathrm{~m}^{-1}$ was measured from the force-distance curve in contact mode using a silicon wafer. The cantilever stiffness $k=1.35 \mathrm{~N} / \mathrm{m}$ was measured by the Thermal $K$ method [16,17], implemented in the PicoView software. Finally, the tip radius $R=1.3 \pm 0.1 \mathrm{~nm}$ 
was estimated from the capillary force $F_{c}$ when the tip was brought into contact with water [18]: $R \approx F_{c} /(4 \pi \gamma)$, where $\gamma \approx 70 \mathrm{~mJ} \mathrm{~m}^{-2}$ is the surface tension of water.

\section{Nanomechanics}

After the samples had been imaged in contact mode, the force-displacement curves were measured on the region of interest in and around a pit. The vertical position of the tip was known from the calibrated voltage applied to the $z$ piezotransducer, and the cantilever deflection was determined from the output voltage $V_{A-B}$ of the photodetector. Each experiment was repeated at least ten times on the same pit and for ten different pits of two different samples. We carried out two series of measurements: one on a set of dried samples, the other on a set of fully water-saturated samples.

The local mechanical properties of the pits were calculated from the force-displacement curves. For Hertzian indentation, the raw $V_{A-B}$ data are related to the indentation $\delta$ by a power law:

$$
V_{A-B}=\frac{4}{3} \frac{\left(R^{1 / 2} E\right)}{k \chi} \delta^{3 / 2},
$$

where $E$ is the indentation modulus. Assuming that the Young's modulus of the tip is high $\left(E_{\text {tip }}=168 \mathrm{GPa}\right.$ [19] $)$ and neglecting the effect of the Poisson ratio, $E$ represents the Young's modulus of the sample. The displacement $\delta$ was obtained by subtracting values obtained at the same force for the tip displacement of the sample and the tip displacement of the silicon wafer. $E$ can be calculated from the slope of the experimental curve of $V_{A-B}$ plotted against $\delta^{3 / 2}$ according to Eq. (2). Indentation experiments on model materials have shown that the $z$ piezo-transducer should be calibrated. We used polymethyl methacrylate (PMMA), which has a Young's modulus close to that of the wood $\left(E_{\mathrm{PMMA}}=2.1 \mathrm{GPa}[20]\right)$ in order to determine the calibration factor. The force displacement curves of PMMA and silicon wafer, and the indentation curve $V_{A-B}$ plotted against $\delta^{3 / 2}$ for PMMA, are presented in Figs. 2(a) and 2(b), respectively.

Assuming that the pit membrane is a thin embedded circular plate, loaded by a point force at its center, the deflection $z$ at the center is [21]

$$
z=\frac{F L^{2}}{4 E e^{3}}\left[\frac{3(2-v)\left(1-v^{2}\right)}{\pi}\right],
$$

where $F$ is the force at the center, $L$ is the diameter of the plate, $e$ is the plate thickness, and $v$ is the Poisson ratio. Note that the tip displacement is proportional to the applied force. The Young's modulus of the membrane, $E$, is calculated from the slope of $V_{A-B}$ plotted against $z$.

\section{Air seeding}

We developed an experimental method (Fig. 3) to measure the critical pressure difference $\Delta P_{c}$ that allowed air to flow into the xylem system. This pressure difference is an intrinsic property of the trees [22]. One end of a segment of a Populus branch was connected to a bottle of compressed air by means of a special airtight clamp; the other end was immersed in a water receptacle. The pressure in the air injected into the end of the branch was controlled by a pressure transducer.

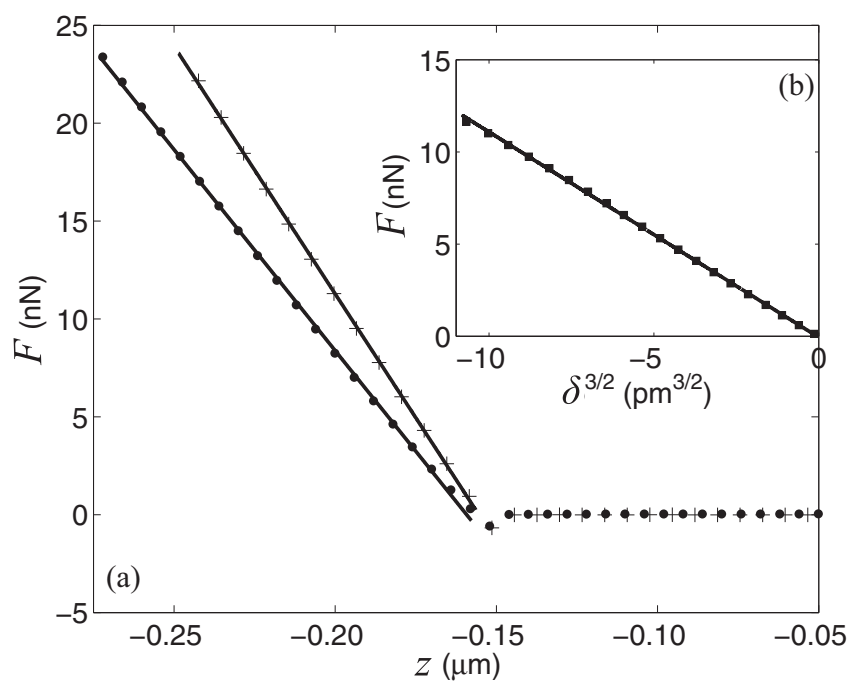

FIG. 2. (a) Force-displacement curves for PMMA (•) and for silicon wafer $(+)$. The continuous lines $(-)$ display the linear fit, the calibration factor is $1 / \chi=2.89 \pm 0.1410^{8} \mathrm{~V} / \mathrm{m}$. (b) Nanoindentation curve of PMMA (ם) with its linear fit (-).

The pressure difference between the two ends of the branch segment was varied between 0.1 and $3 \mathrm{MPa}$ with an accuracy of $50 \mathrm{kPa}$. After each pressure increase, we verified that the pits were undamaged by measuring a zero air flow rate at $0.2 \mathrm{MPa}$ during $3 \mathrm{~h}$.

The flow rate $Q_{T}$ was determined for different $\Delta P$ between $\Delta P_{c}$ and $3 \mathrm{MPa}$. Flow rates were determined in the steady state by measuring the height of the water column in a calibrated test tube that was placed in the water receptacle. The flow rate corresponding to a given pressure difference was obtained as the average of 5 repetitions with 12 different branches. The permeability $K$ of the samples was determined from the Darcy law:

$$
K=\frac{Q_{T} \mu}{A} \frac{L_{b}}{\Delta P},
$$

where $\mu$ is the air viscosity, $\Delta P$ is the pressure difference, $L_{b}$ is the branch length, and $A$ is the area of the branch section. Note that the bark serves as a natural impermeable seal on the outer cylindrical surface of the branch.

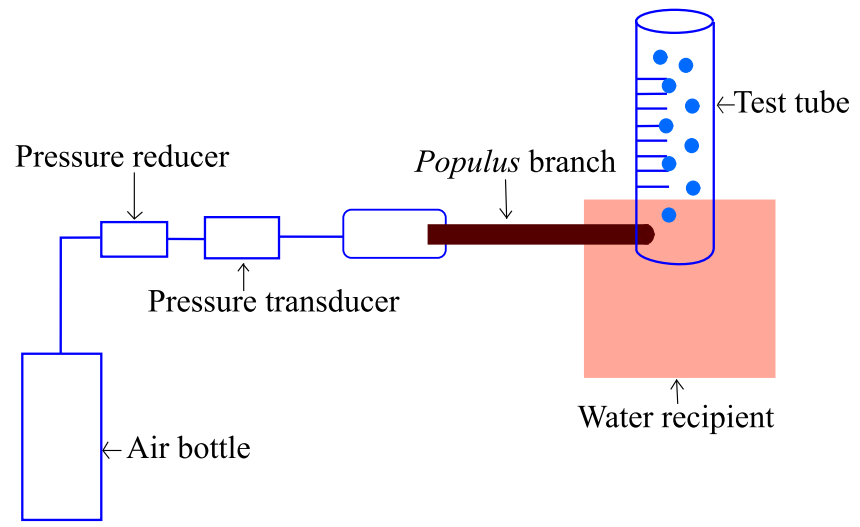

FIG. 3. (Color online) Diagram of air seeding experiments. 


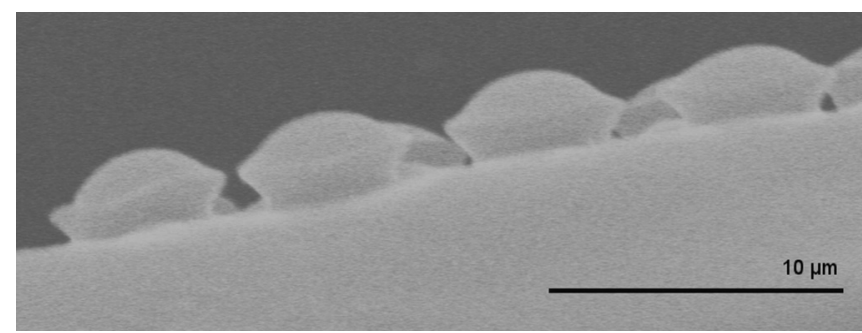

FIG. 4. Silicone micromolding of Populus pits at $\Delta P=2.2 \mathrm{MPa}$ observed by SEM.

\section{Micromolding}

The sample extremities were first trimmed with a razor blade to ensure that the vessels were open. Stems were cut under water to avoid native embolism. All bark material was removed before experiments. We used the silicone molding liquid RTV 141, which is stable against the chemical reagents used for digestion of organic materials. Furthermore, the silicone does not diffuse through the pit membranes [23]. The RTV 141 silicone is a two-component material. The two components were degassed for $2 \mathrm{~h}$ before use. The injection system consisted of a pressure chamber supplied by a compressed air bottle. One end of a branch was placed in the pressure chamber in contact with the silicone, the other end remaining outside the chamber at atmospheric pressure. The injection pressure in the chamber was established gradually. The silicone fluid flowed into the xylem until it was stopped by the pits. These experiments were made with two different branches at three different injection pressures: $\Delta P=0.2 \mathrm{MPa}$, $\Delta P=\Delta P_{c}$ and $\Delta P=2.2 \mathrm{MPa}$. After $3 \mathrm{~h}$ of crosslinking under pressure, the specimens were annealed at $50{ }^{\circ} \mathrm{C}$ for $3 \mathrm{~h}$ at atmospheric pressure to complete the chemical reaction. Finally, all biological material was removed by immersing the sample in a Franklin's solution and a sulfuric acid solution for $24 \mathrm{~h}[24,25]$. The silicone moldings of the vessels were subsequently observed by optical microscopy and by SEM (Fig. 4).

The pit membrane deformations were measured by analysis of the SEM images. The Young's moduli of the membranes were obtained by fitting the pit membrane deflection to the theoretical prediction Eq. (6) using Matlab and COMSOL Multiphysics software [26].

\section{RESULTS AND DISCUSSION}

\section{A. Structure of the microfluidic system}

The microfluidic system of xylem vessels can be considered as an array of long, parallel capillaries. The mean diameter of these capillaries measured by AFM was $d_{\text {cap }} \simeq 20 \mu \mathrm{m}$ (Fig. 5). The maximum number of xylem vessels in the cross section of the branches that were studied was therefore $\approx 2 \times 10^{4}$. Xylem vessels are connected to each other by pits. Optical microscopy in fluorescence mode allowed the distribution of pits to be determined: the intervascular pits were grouped together at the ends of the vessels. In the intervessel pit field [Fig. 5(a)], adjacent pits were separated by a distance approximately $0.28 \mu \mathrm{m}$. The number of pits at the end of a vessel was estimated to be $84 \pm 10$. The
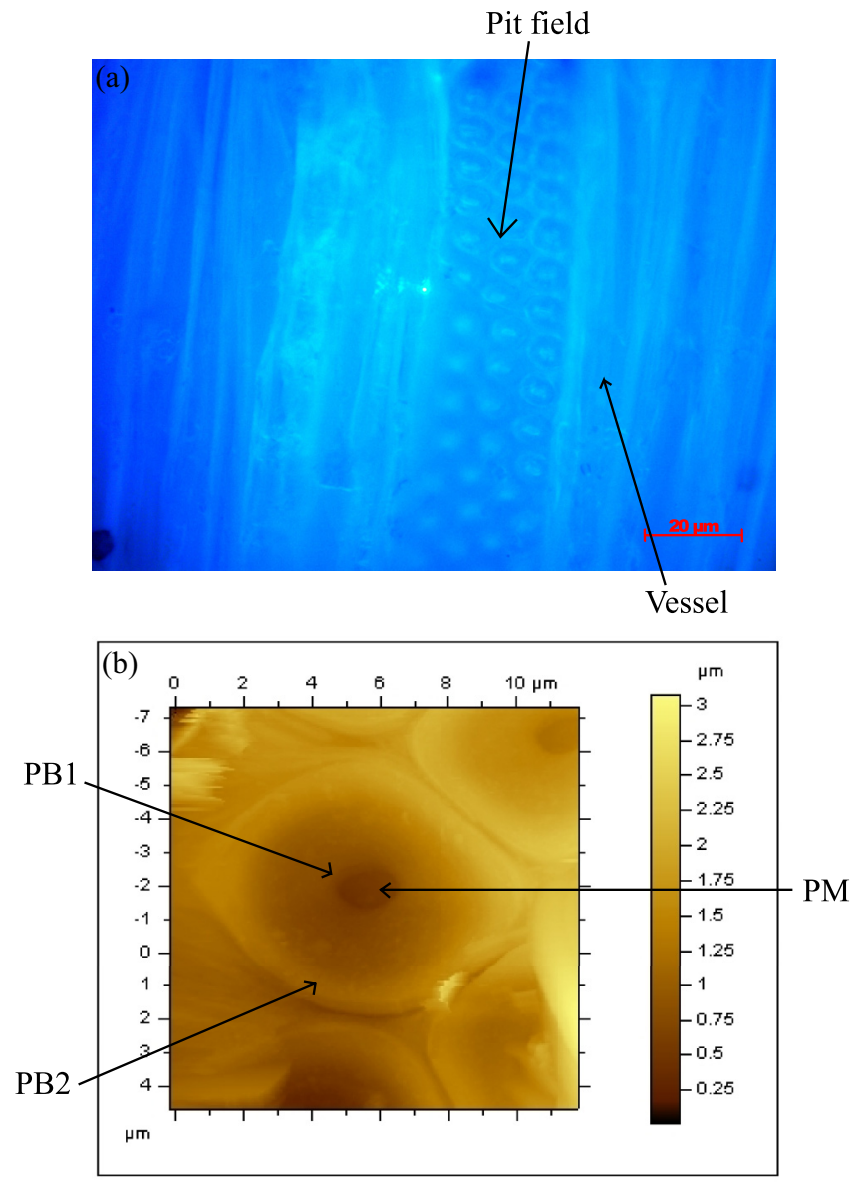

FIG. 5. (Color online) (a) Optical observation of a primary pit field $(\times 100)$. (b) Topography image of a dried Populus pit measured by AFM in tapping mode.

mean pit diameter determined from AFM observations was approximately $7 \mu \mathrm{m}$. These measurements agree with those from TEM observations that gave a mean diameter $d_{\text {pit }}=$ $8.39 \pm 0.9 \mu \mathrm{m}$ (Fig. 6). The average pit membrane thickness $e=310 \pm 90 \mathrm{~nm}$ was measured only by TEM. TEM shows that the thin pit membrane is attached at the bottom of the at the junction between the two pit borders. When viewed by AFM, only the central part of the pit membrane is visible, since an annular structure, the pit border, with a mean aperture diameter of approximately $1.5 \mu \mathrm{m}$, covers it partially as seen in Fig. 5(b). Two characteristic regions can be defined [Figs. 5(b) and 6]: the pit border extremity near the pit aperture, denoted $\mathrm{PB} 1$, and the pit border near the intravascular pit junctions, denoted PB2.

AFM only allows us to observe the pit membrane in the center of the pit. Figure 5(b) shows the topography image of a dried Populus pit. There are no regular surface structures, on either the dried pit membrane or the pit border. Indeed, we cannot detect the presence of microfibrils that were observed on Sapium pits by AFM by Pesacreta et al. (2005) [10].

\section{B. Nanomechanics of pits}

Force-displacement curves for dried samples were obtained by AFM. Our aim was to characterize the nanomechanical 


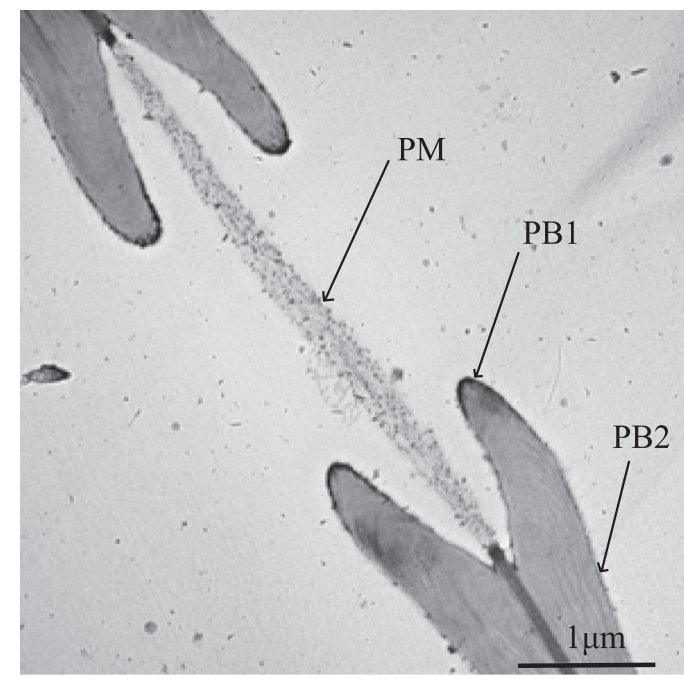

FIG. 6. TEM observation of a dried intervascular Populus pit. The pit membrane (PM) thickness is around $311 \mathrm{~nm}$. The pit border (PB) partially covers the pit membrane.

properties of the vessel wall, PB1, PB2, and the pit membrane (PM).

Example force-displacement curves for the vessel wall are presented in Fig. 7(a). After measuring with the same tip the force-displacement curve of a silicon wafer, we calculated the indentation $\delta$ and verified that $V_{A-B}$ was proportional with $\delta^{3 / 2}$ [Fig. 7(b)]. We observed that the adhesion force between the tip and the sample can be neglected ( $F \simeq 0$ for $\delta \simeq 0$ ). From these experiments, we found that the Young's modulus of the vessel wall is $E=7.9 \pm 0.4 \mathrm{GPa}$. This value is in agreement with the literature [27] and corresponds to the radial Young's modulus of cell walls. Similarly, we measured the force-displacement curves of PB1 and PB2. However, we did not find a model to fit the raw data and from which we could extract the Young's

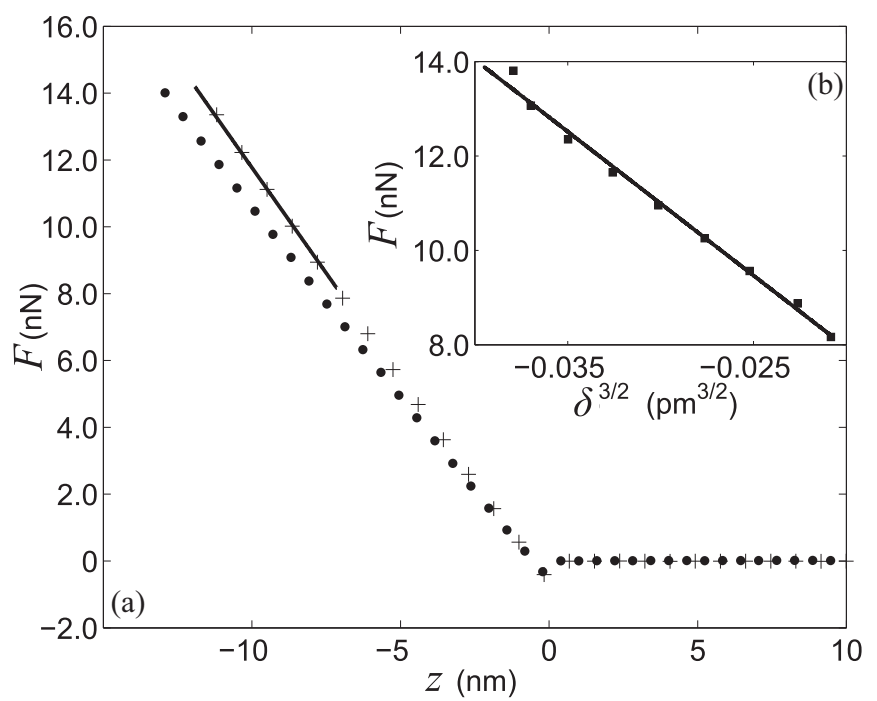

FIG. 7. (a) Force-displacement curves on a xylem vessel wall (•) and on a silicon wafer $(+)$. (b) Nanoindentation curve on a xylem vessel wall (ם) with its linear fit (-).

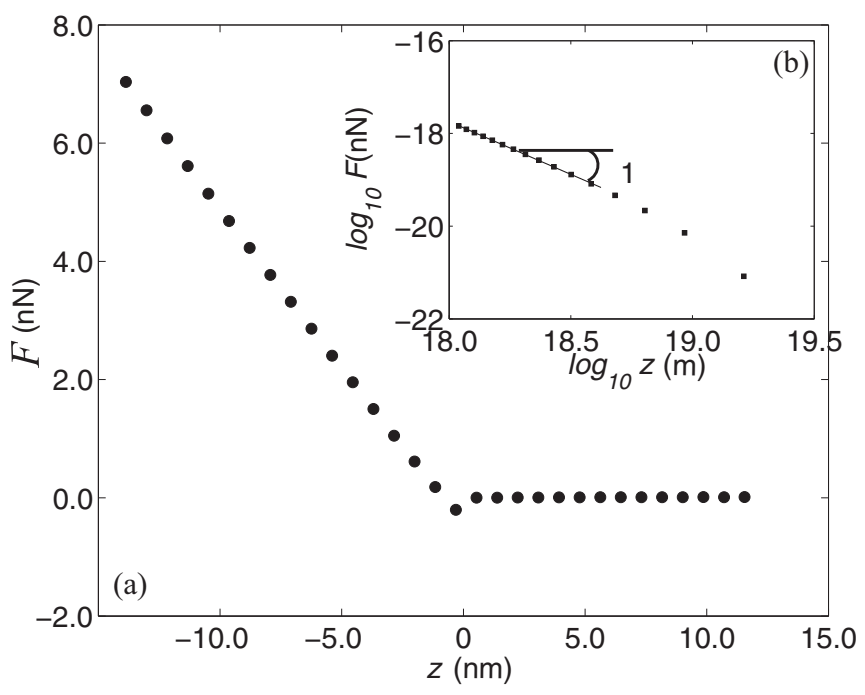

FIG. 8. (a) Force-displacement curve on a pit membrane (PM) (•). (b) Log-log representation of the force-distance curve of the pit membrane (ם) with its linear fit (-).

modulus. This is due to the fact that the pit border moves under the effect of the tip force.

The force-distance curve of the pit membrane is plotted in Fig. 8(a). In this example, the strength was proportional to the tip displacement [Fig. 8(b)]. This behavior is characteristic of a flexion test and the Young's modulus $E=0.36 \pm 0.02 \mathrm{GPa}$ was calculated from Eq. (3). Thus, at a given pressure difference, the strain in the pit membrane is much higher than that in the vessel wall.

Nanoindentation experiments were performed on PM, PB1, and PB2 in water saturated samples. The force-displacement curves [Fig. 9(a)] were characteristic of a viscoelastic solid material. At low forces, the curves were fitted by Zener rheological model [Fig. 9(b)], for which the stress-strain

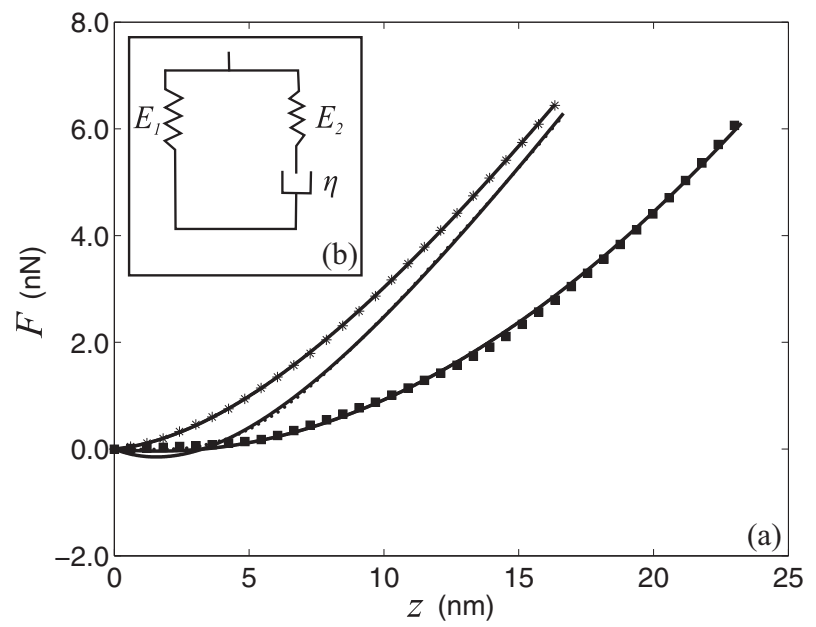

FIG. 9. (a) Force-displacement curves for water-swollen pit membranes (PM) $(\bullet)$, pit border extremity (PB1) (ם), and pit border near the intravascular pit junctions (PB2) $(*)$ fitted by a Zener model (-). (b) The Zener model. 
TABLE I. Zener elements of swollen pit membrane (PM), pit border extremity (PB1), and pit border near the intravascular pit junctions (PB2) determined by nanoindentation experiments of swollen pits. The values of the Zener elements are given with an accuracy of $15 \%$.

\begin{tabular}{llll}
\hline \hline Elements & PM & PB1 & PB2 \\
\hline$E_{1}(\mathrm{GPa})$ & 0.41 & 1.66 & 0.43 \\
$E_{2}(\mathrm{GPa})$ & 0.95 & 3.35 & 0.84 \\
$\tau(\mathrm{s})$ & 0.99 & 63.3 & 6.2 \\
\hline \hline
\end{tabular}

relationship $\sigma(\epsilon)$ is

$$
\sigma=E_{1} \epsilon+\eta \dot{\epsilon}\left[1-\exp \left(-\frac{\epsilon}{\tau \dot{\epsilon}}\right)\right],
$$

where $\tau$ is the relaxation time, $\dot{\epsilon}$ is the strain rate, $\eta$ is the viscosity, and $E_{1}$ and $E_{2}$ are the Young's moduli. In our experiments, $\dot{\epsilon}$ was defined as a constant tip velocity normalized by the tip radius: $\dot{\epsilon}=1.17 \pm 0.06 \mathrm{~s}^{-1}$. In the Zener model, $E_{1}$ corresponds to the low frequency Young's modulus of the specimens and $E_{1}+E_{2}$ to the high-frequency modulus. We observed a very good agreement between the model and the experimental data. Table I reports the values of the Zener elements obtained from the force-displacement curve fits. These values were obtained using the Matlab software by minimization of the standard deviation. PM and PB2 are characterized by similar Zener elements. On the other hand, PB1 showed higher moduli and a larger relaxation time. However, all the values are close and indicate that the three analyzed areas have a similar chemical composition. Finally, for PM, the comparison of the Young's modulus $E$ of dried samples with the Young's modulus $E_{1}$ of water-saturated samples showed that the PM mechanical properties were not affected by the sorption of water.

\section{Air microfluidics}

From air-seeding experiments, we found that the critical air pressure difference $\Delta P_{c}$ at which nonzero flow first occurred was around $1.80 \pm 0.02 \mathrm{MPa}$ for 50 Populus branches (Fig. 10). We verified that air had entered the branches, which were now only partially filled with water. Hence, $\Delta P_{c}$ should be the average critical pressure that causes a single meniscus within a pore to break. At $\Delta P_{c}$, the flow rate $Q_{T}$ jumped from zero to a value typically around $10^{-8} \mathrm{~m}^{3} \mathrm{~s}^{-1}$ and we observed by optical microscopy at the outlet end of the branch segment that a very low number of xylem vessels $\left(n_{\text {cap }} \approx 10\right)$ were involved in the air flow. Indeed, the air conductive vessels are mainly linked to the wood knots that is involved in the main part of the air flow. So, most of the flow rate is dedicated to the development of ramifications and buds. The pressure dependence of the air flow rate $Q_{T}$ for $\Delta P \geqslant \Delta P_{c}$ is presented in Fig. 10. We observe that $Q_{T}$ varies between $1.29 \pm 0.0610^{-8}$ and $4.78 \pm 0.0610^{-8} \mathrm{~m}^{3} \mathrm{~s}^{-1}$ when $\Delta P$ changes from 1.8 to $2.8 \mathrm{MPa}$. The air permeability $K$ at the critical pressure $\Delta P_{c}$ calculated from Eq. (4) is $1.6 \pm 0.110^{-15} \mathrm{~m}^{2}$.

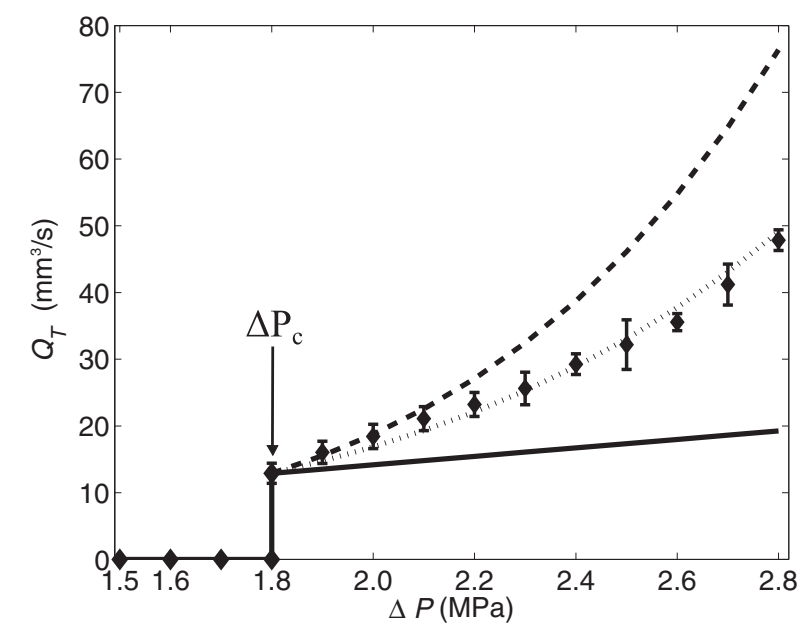

FIG. 10. Experimental air seeding flow rate vs. difference pressure $(\checkmark)$. Modeling of the flow rate at constant number of pit membrane pores: (-) pressure effects, (- - -) pressure effects combined with the pit membrane deformation ( $E=0.41 \mathrm{GPa})$, and $(\cdots)$ pressure effects combined with the pit membrane deformation $(E=0.65 \mathrm{GPa})$.

If the pit membrane is assumed to be a simple circular plate with clamped edges, the linear elastic deflection $z(r)$ of the membrane under a differential pressure $\Delta P_{c}$ is [26]

$$
z(r)=\frac{12\left(1-v^{2}\right)}{64} \frac{\Delta P_{c}\left(R_{\mathrm{pit}}^{2}-r^{2}\right)^{2}}{E e^{3}},
$$

where the pit radius $R_{\text {pit }} \simeq 3.5 \mu \mathrm{m}$, the pit membrane thickness $e \simeq 310 \mathrm{~nm}$, and the Poisson ratio $v \simeq 0.23$ [28]. Using these geometrical values, the maximum pit membrane strain was around $84 \%$ [26].

In this natural system, three intrinsic properties have been brought to light: the membrane deformation, the critical pressure, and the fact that we can assume that the sap is biologically and chemically inert [7]. Moreover, the microsystem works not only under a positive pressure (in our experiments) but also under tension (in vivo). The behavior of the system is in both cases the same. So the mechanism of air diffusion must be passive. The only explanation of these three properties is that the membrane is porous. The critical pressure difference, $\Delta P_{c}$, characteristic of the pit porosity, was then defined as the minimum pressure that allowed the air flow through the branch. Water menisci are present in pit membrane pores and prevent any air flow until a critical pressure at which the menisci break. In this way, the pit membranes control the flow of air, acting as capillary valves that open at $\Delta P_{c}$. This pressure difference varies inversely with the pore diameter $d_{\text {pore }}$, as given by the Laplace relation Eq. (1). In this relation, the contact angle between the vapor interface and the pit membrane, $\theta$, is assumed to be $0^{\circ}$ [11]. Recent measurement of the capillary pressure difference in angiosperm species concluded that the pit membrane pore diameter should range from 100 to $200 \mathrm{~nm}$ [29,30]. On the other hand, experiments with colloidal gold seeding particles indicated that the pore diameter of the pit membrane varies between 5 and $20 \mathrm{~nm}$ [31]. These different results can be understood by allowing for the fact that the pit membrane is thin and can be strained under 
the capillary pressure difference, increasing in this way the pore diameter [29]. Moreover, the vulnerability to embolism seems to depend on the ratio between the pit chamber depth and the pit membrane diameter: high embolism sensitivity is correlated with a high value of this ratio.

We model the pit membrane as a porous medium of $n_{\text {pore }}$ pores of diameter $d_{\text {pore }}$ and length $e$. The flow rate $Q_{\text {pore }}$ in one pore is given by the Poiseuille relation:

$$
Q_{\text {pore }}=\frac{\pi}{128 \mu} \frac{\Delta P}{e} d_{\text {pore }}^{4},
$$

where $\Delta P / e$ is the pressure gradient.

Here, we assumed that the gas is compressed in one cell, flows in the pores, and undergoes an isenthalpic expansion in the adjacent cell (the estimated Mach number is close to 0.7). Based on the assumption that the hydraulic pressure drop in the vessel is negligible compared with the pressure drop across the pit membrane, the total flow rate in the branch segment $Q_{T}$, which contains $n_{\text {cap }}$ conducting capillaries in parallel and a number of pits $\left(n_{\text {pit }}\right)$ per capillary, is [32]

$$
Q_{T}=n_{\text {cap }} n_{\text {pit }} n_{\text {pore }} \frac{\pi d_{\text {pore }}^{4} \Delta P}{128 \mu e} .
$$

The total flow rate can also be expressed as:

$$
Q_{T}=n_{\text {cap }} Q_{\text {cap }},
$$

where $Q_{\text {cap }}$ is the flow rate in one capillary given by the relation

$$
Q_{\text {cap }}=\frac{\pi}{128 \mu} \frac{\Delta P}{L_{b}} d_{\text {cap }}^{4} .
$$

From Eqs. (4) and (9), we found the relationship between $K$ and $n_{\text {cap }}$ :

$$
n_{\text {cap }}=\frac{128 K A}{\pi d_{\text {cap }}^{4}},
$$

which is an approximation of the number of conducting xylem vessels. Equations (8) and (11) are established on the basis of three main assumptions:

(1) The vessel walls are air proof and waterproof.

(2) The branch is modeled as a porous medium. The flow rate follows the Poiseuille's law, which is a function of the average number of conducting pores. The pores have a constant mean diameter, $d_{\text {pore}}$, and a mean length close to the pit membrane thickness, $e$.

(3) The flow rate is also calculated from the value of the average number of conducting vessels per branch, $n_{\text {cap }}$ [determined by Eq. (9)], from the average number of pits per vessel, $n_{\text {pit }}$ (determined by optical microscopy), and from the average number of conducting pores per pit, $n_{\text {pore }}$ (estimated from the fit of the experimental data). Hence, the product $n_{\text {cap }} n_{\text {pit }} n_{\text {pore }}$ gives the average number of conducting pores per branch.

Hence, for $\Delta P=\Delta P_{c}$, the pit membrane pores were dilated. The mean diameter of these pores was estimated from Eq. (1) using a contact angle $\theta=0: d_{\text {strained pore }} \simeq 160 \mathrm{~nm}$. This value is in agreement with those obtained in the literature [29]. Finally, considering the membrane deformation, we can also estimate that the pore diameter of the unstrained pit membrane is close to $49 \mathrm{~nm}$. This value is an average pore diameter
TABLE II. Pit membrane properties: $d_{\text {pore }}$ is the pore diameter of unstrained pit membrane; $d_{\text {strained pore }}$ is the strained pore diameter of the pit membrane for $\Delta P_{c}=1.8 \mathrm{MPa}$ measured by air seeding experiments; $e$ is the pit membrane thickness; $n_{\text {pore }}$ is the number of conducting pores in the pit membrane; $K\left(\Delta P_{c}\right)$ is the air permeability of the sample for $\Delta P_{c} ; E$ is the Young's modulus of dried pit membrane.

\begin{tabular}{lc}
\hline \hline Pit membrane properties & \\
\hline$d_{\text {pore }}(\mathrm{nm})$ & $49 \pm 5$ \\
$d_{\text {strained pore }}(\mathrm{nm})$ & $160 \pm 8$ \\
$e(\mathrm{~nm})$ & $310 \pm 90$ \\
$n_{\text {pore }}$ & $4 \pm 1$ \\
$K\left(\Delta P_{c}\right)\left(\mathrm{m}^{2}\right)$ & $1.6 \pm 0.110^{-15}$ \\
$E(\mathrm{GPa})$ & $0.36 \pm 0.02$ \\
\hline \hline
\end{tabular}

within the pit membrane population (which has a large distribution of sizes, $d_{\text {pore }}$ is assumed to follow a Gaussian distribution, as expected for polymeric systems [33]). In order to interpret the air-seeding results, we first calculated, from Eq. (9), the number of xylem vessels $n_{\text {cap }} \approx 6$ that provide the flow rate for $\Delta P_{c}$. This value is close to that obtained from optical observations. Figure 10 compares the flow rate modeled by a simple increase of pressure, Eq. (8), with the flow rate modeled by an increase of pressure combined with the pit membrane deformation by considering that the pore diameters grow with the pit membrane deflection, assuming constant $n_{\text {cap }}=6$. We observe that the experimental curve is located between these two modeled curves. These results show clearly that the observed nonlinear pressure dependence of flow rate cannot be explained by the model with a constant pore diameter. On the other hand, if we take into account the free pit membrane deformation, the calculated flow rate is systematically overestimated. Based on this analysis, we conclude that the pit membrane deformation may be limited by the pit border, restricting the pore opening. The pit border reduces the deflection of the membrane and limits the strain since the bending can only occur in the pit aperture. The pit membrane deflection in presence of the pit border can be modeled by Eq. (6) with a higher Young's modulus. Indeed, COMSOL modeling showed that the pit membrane deformation when constrained by the pit border is similar to the pit membrane deformation without the pit border and with a higher Young's modulus. Figure 10 shows that a pressure increase combined with a pit membrane deflection corresponding to $E=0.65 \mathrm{GPa}$ allows a good fit of the experimental air-seeding curve to be obtained. We verified that $Q_{T} / d_{\text {pore }}^{4} \Delta P \sim$ cst in the accuracy of the experiments. The mean number of conducting pores, $n_{\text {pore }} \approx 4$, can be estimated from Eq. (8). This mean value probably hides a large variability of $n_{\text {pore }}$ between different pits. The pit properties are reported in Table II.

\section{Micromolding}

Figure 11 compares the deformation of three pit membranes under pressure differences of $0.2,1.8$, and 2.2 $\mathrm{MPa}$. The deflection profiles obtained from the SEM images were fitted by Eq. (6), taking the Young's modulus to be an adjustable 

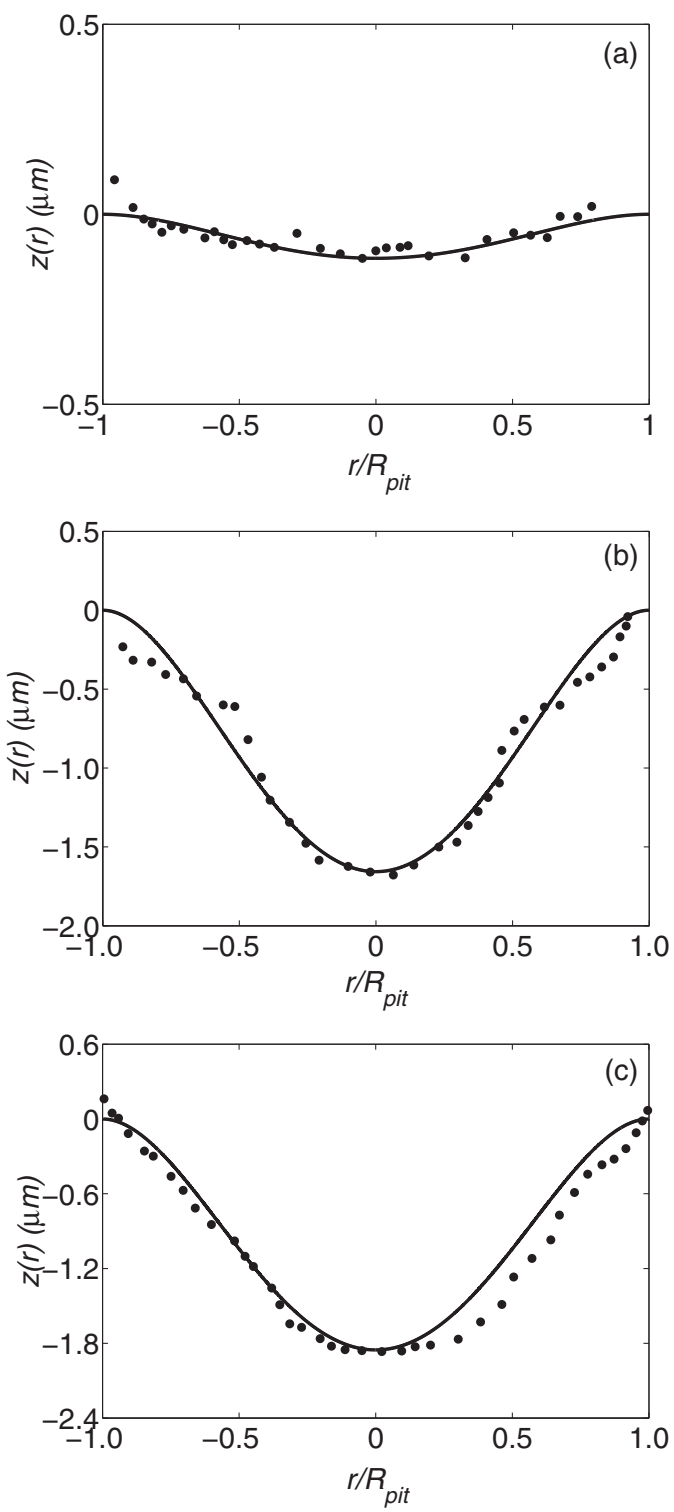

FIG. 11. Deflection of the pit membrane as a function of the normalized radial position $r / R_{\text {pit }}$, measured from micromolding experiments by SEM image analysis at $\Delta P=0.2 \mathrm{MPa}$ (a), $\Delta P=$ $\Delta P_{c}$ (b) and $\Delta P=2.2 \mathrm{MPa}$ (c). The profiles were fitted by Eq. (6) (-) with $E=0.41 \pm 0.15 \mathrm{GPa}$ (a), $E=0.41 \pm 0.13 \mathrm{GPa}$ (b), and $E=0.66 \pm 0.097 \mathrm{GPa}(\mathrm{c})$.

parameter. We found $E=0.41 \pm 0.15 \mathrm{GPa}$ at $\Delta P=0.2 \mathrm{MPa}$; $E=0.41 \pm 0.13 \mathrm{GPa}$ at $\Delta P=\Delta P_{c}=1.8 \mathrm{MPa}$, and $E=$ $0.66 \pm 0.097 \mathrm{GPa}$ at $\Delta P=2.2 \mathrm{MPa}$. These results support the idea that the pit membrane are strained under the pressure difference and are limited by the pit border for $\Delta P>\Delta P_{c}$. The Young's modulus values measured at $\Delta P=2.2 \mathrm{MPa}$ is identical of that deduced from Fig. 10. Finally, these results validated the measurement of the pit membrane mechanical properties by AFM.

\section{CONCLUSION}

We have studied the flow of air in the Populus xylem, which is a natural microfluidic system. Our aim was to understand the mechanism by which flow of air within the plant is controlled, in order to develop new MEMS biomimetic devices. We first characterized the structure of the natural microfluidic network by optical, electronic, and atomic force microscopy. The vessels are connected by typically a hundred pits, formed of a thin membrane of thickness $310 \mathrm{~nm}$. We measured the mechanical properties of the vessel wall and of the pit by AFM, and found that the pit membrane has Young's modulus close to $0.4 \mathrm{MPa}$. Air seeding experiments showed that the threshold pressure difference at which air can flow when the microfluidic system is only partially saturated with water is $\Delta P_{c}=1.8 \mathrm{MPa}$. Micromolding experiments showed that the pit membrane is strained by the applied pressure difference, with a typical maximum strain around $80 \%$ at $\Delta P_{c}$. Furthermore, these experiments established that the pit borders limit the pit membrane deformation for $\Delta P>\Delta P_{c}$. We have also verified that the Young's modulus deduced from the SEM image analysis of silicone micromoldings is in agreement with the value measured by AFM. Finally, we have measured the air flow rate for $\Delta P \geq \Delta P_{c}$ by air seeding experiments. We propose that the flow of air can be modeled by considering that pits behave as porous membranes which become strained under the applied pressure difference. All these results support the idea that the air flow in the Populus xylem is controlled by a system of capillary valves. These valves are formed of water menisci in the pit membrane pores, and $\Delta P_{c}$ is the value of the capillary pressure difference at which the menisci break. Any increase in the pressure difference across the membrane increases the pore diameter due to deformation of the pore membrane, thereby reducing $\Delta P_{c}$. In the future, this system of capillary valves could be used for the development of new MEMS, for biological or medical applications.

\section{ACKNOWLEDGMENT}

The authors thank Gregory Ehses for his help in the construction of the air-seeding device and John Sherwood for fruitful discussions.
[1] M. H. Zimmermann and A. A. Jeje, Can. J. Bot. 59, 1882 (1981).

[2] S. Jansen, B. Choat, and A. Pletsers, Am. J. Bot. 96, 409 (2009).

[3] M. T. Tyree and M. H. Zimmermann, Xylem Structure and the Ascent of Sap, 2nd ed. (Springer-Verlag, Berlin, 2001).

[4] M. T. Tyree and J. S. Sperry, Annu. Rev. Plant Phys. Mol. Biol. 40, 19 (1989).
[5] T. D. Wheeler and A. D. Stroock, Nature 455, 208 (2008).

[6] O. Vincent, P. Marmottant, P. A. Quinto-Su, and C.-D. Ohl, Phys. Rev. Lett. 108, 184502 (2012).

[7] A. D. Stroock, V. V. Pagay, M. A. Zwieniecki, and N. M. Holbrook, Annu. Rev. Fluid Mech. 46, 615 (2014).

[8] A. G. Meyra, V. A. Kuz, and G. J. Zarragoicoechea, Tree Physiol. 27, 1401 (2007). 
[9] J. Lee, N. M. Holbrook, and M. A. Zwieniecki, Front. Plant Sci. 3, 55 (2012).

[10] T. C. Pesacreta, L. H. Groom, and T. G. Rials, IAWA J. 26, 397 (2005).

[11] P. G. de Gennes, F. Brochard-Wyart, and D. Quéré, Capillarity and Wetting Phenomena: Drops, Bubbles, Pearls, Waves (Springer, New York, 2004).

[12] G. Binnig, C. F. Quate, and Ch. Gerber, Phys. Rev. Lett. 56, 930 (1986).

[13] S. S. Nair, S. Wang, and D. C. Hurley, Composites 41, 624 (2010).

[14] W. C. Oliver and G. M. Pharr, J. Mater. Res. 7, 1564 (1992).

[15] O. Piétrement and M. Troyon, J. Colloid Interf. Sci. 226, 166 (2000).

[16] B. Capella and G. Dietler, Surf. Sci. Rep. J. 34, 1 (1999).

[17] J. N. Sharpe, Handbook of Experimental Solid Mechanics (Springer, Berlin, 2008).

[18] J. Crassous, E. Charlaix, H. Gayvallet, and J. L. Loubet, Langmuir 9, 1995 (1993).

[19] R. J. Jaccodine, J. Electrochem. Soc. 110, 524 (1963).

[20] E. Amitay-Sadovsky and H. D. Wagner, Polymer 39, 2387 (1998).

[21] J. G. Williams, Stress Analysis of Polymers (Ellis Horwood, Hempstead, UK, 1980).

[22] H. Cochard, P. Cruiziat, and M. T. Tyree, Plant Physiol. 100, 205 (1992).
[23] J. P. André, Organisation vasculaire des angiospermes: une vision nouvelle (Quae, Versailles, France, 2002).

[24] J. S. Sperry, U. G. Hacke, and J. K. Wheeler, Plant Cell Environ. 28, 456 (2005).

[25] J. K. Wheeler, J. S. Sperry, U. G. Hacke, and N. Hoang, Plant Cell Environ. 28, 800 (2005).

[26] S. P. Timoshenko and S. Woinowsky-Krieger, Theory of Plates and Shells, 2nd ed. (McGraw-Hill International Editions, New York, 1959).

[27] M. Eder, O. Arnould, J. W. C. Dunlop, J. Hornatowska, and L. Salmén, Wood Sci. Technol. 47, 163 (2013).

[28] D. W. Green, J. E. Winandy, and D. E. Kretschmann, in Wood Handbook: Wood as an Engineering Material, Gen. Tech. Rep. FPL-GTR-113 (U.S. Department of Agriculture, Forest Service, Forest Products Laboratory, Madison, WI, 1999), p. 463.

[29] B. Choat, S. Jansen, M. A. Zwieniecki, E. Smets, and N. M. Holbrook, J. Exp. Bot. 55, 1569 (2004).

[30] M. W. Shane, M. E. McCully, and M. J. Canny, Ann. Bot. 85, 613 (2000).

[31] B. Choat, M. Ball, J. Luly, and J. Holtum, Plant Physiol. 131, 41 (2003).

[32] E. Guyon, J. P. Hulin, L. Petit, and C. D. Mitescu, Physical Hydrodynamics (Oxford University Press, Oxford, 2001).

[33] J. Comyn, Polymer Permeability (Chapman \& Hall, London, 1985). 